Article

\title{
Application of an Improved Formal Model of the Hybrid Development of Ontologies in Complex Information Systems
}

\author{
Yousef Ibrahim Daradkeh ${ }^{1}$ and Iryna Tvoroshenko ${ }^{2, *}$ \\ 1 Department of Computer Engineering and Networks, College of Engineering at Wadi Addawasir, \\ Prince Sattam Bin Abdulaziz University, Al-Kharj 11991, Saudi Arabia; y.daradkeh@psau.edu.sa \\ 2 Department of Informatics, Kharkiv National University of Radio Electronics, 14 Nauka Ave., \\ Kharkiv 61166, Ukraine \\ * Correspondence: iryna.tvoroshenko@nure.ua
}

Received: 7 August 2020; Accepted: 25 September 2020; Published: 27 September 2020

\begin{abstract}
Ontologies in artificial intelligence systems are an effective way to represent and integrate knowledge and data. The property of such structures is that any subject area is accurately described in formal language. There is a problem in the research and determination of the adequacy of ontologies under development. The perspective directions are model construction for the development of fuzzy ontologies and also the creation of methods for evaluating adequacy. The achieved results allow one to implement the processes of supporting the development and integration of ontologies of complex systems on the basis of intelligent approaches. The method is proposed to solve the problem of alternative representation and the integration of knowledge and data in artificial intelligence systems. The methodology of improving the model of the hybrid development of fuzzy ontologies is described here; it provides the preliminary modification of models of extensive and intensive progress of ontologies in space and time. The identified features of fuzzy ontology processing allow us to create a procedure for finding and eliminating inadequacies. The software implementation of the application for the integration and presentation of heterogeneous data is carried out. The consumption of Random Access Memory (RAM) for the proposed models is analyzed. The further perspectives of the proposed research are determined in accordance with the principles of classification.
\end{abstract}

Keywords: ontology; extensive development; intensive development; ontology import relation; adequacy; membership function; computational intelligence

\section{Introduction}

Recent trends have proved that large ontologies are being created as a result of the consolidation of small ontologies, and that their specifications must support more than one standard vocabulary. The consolidation of small ontologies can be carried out manually, but in complex systems an intelligent decision-making system is necessary.

The existing practical results [1-8] show that the achievements of the specified directions do not allow us to develop fully functional intelligent decision-making systems which allow us to combine heterogeneous ontologies and then to eliminate the created conflicts. This is a significant drawback, and in order to eliminate it we should find new approaches for the evaluation and analysis of ontologies. The improvement of intelligent methods for the development of ontologies, mechanisms for inadequacy elimination, and increasing the reliability of managerial decisions according to these approaches are relevant trends in the area of computational intelligence during the design of information systems $[9,10]$.

The methodological aspects of the construction of a formal model of intensive ontology development are presented in [11]. This article does not contain one of the important components of 
the research on ontological systems-namely, the model of extensive ontology development. There is also no practical implementation of the proposed model of intensive ontology development.

The literary sources [10,12-16] show the possibilities and efficiency of using the apparatus of fuzzy logic in intelligent systems. The practical application of the method of fuzzy analytical hierarchy is described in detail in the article [17]. The achieved results of the successful implementation of fuzzy tools can be useful for improving fuzzy ontological models which are able to develop in time and space. There is no description of the ontological representation and application of fuzziness in the ontological systems in [10,12-17].

It should be noted that in the works $[5,18]$ the concepts of the terminology hierarchy, consisting of a set of specific concepts, and the relations hierarchy, consisting of a set of certain relations, are not separated; they represent the specified hierarchy through the consolidation of these two sets.

The analyzed scientific and practical literary sources [1-9,18-21] showed that the problem of ontology research is relevant and requires further expansion and improvement. It is necessary to apply a systematic approach to solve the modified ontological tasks. The perspective direction is the improvement of models of the extensive and intensive development of ontologies, which allow us to organize and justify these processes using computational intelligence as well as the effective integration of the proposed ontologies of complex systems.

There are many tools that allow one to manipulate ontologies in different ways $[1-3,5,7,8]$. However, none of them fully solve the aspects of inadequacy occurrence, in particular inconsistency, in the development process, and this is a significant disadvantage. Thus, the provision of appropriate intellectual means will allow us to raise the work of the specialists involved in the collective processing of ontologies to a new level.

The purpose of recent research is to improve models of the extensive and intensive development of fuzzy ontologies using computational intelligence, along with the simultaneous elimination of the inadequacy that can occur during the evolution of ontologies.

The objects of this research are fuzzy ontological models with the ability to develop in time and space. The subject of the proposed research is the existing model of fuzzy ontologies of complex systems.

As a part of this task, it is necessary to analyze existing solutions to generalize approaches for building fuzzy ontologies. Due to the magnitude of the task and the heterogeneity of the research object, one restriction has been introduced: ontologies under research have an expressiveness level, which reduces to computational descriptive logic. This restriction narrows the field of the application of practical results, but does not reduce the significance and relevance of this research.

The article offers and demonstrates:

- Building the hybrid mathematical model of ontology development, which integrates extensive and intensive models which describe the evolution in space and time, respectively (Sections 3 and 4). The improved model allows us effectively to solve the tasks of analyzing and evaluating the space of states of analyzed ontologies, as well as to determine, localize, and eliminate the inadequate properties;

- The method for eliminating ontologies' inadequate properties (Section 5), which uses formal criteria for incompleteness and the redundancy of inconsistency, allows us to solve tasks in a fuzzy metric space, increasing the probability of the decisions taken;

- Algorithmic and software tools for eliminating inconsistency (Sections 5 and 6), obtained as a result of the implementation of the method to eliminate ontological information inadequate properties.

Thus, this article proposes the principles of intelligent tool construction for the task of ontology development, which allow us to solve the tasks of searching and localizing the facts of inadequacy during the evolution of ontologies. 


\section{Background}

Modern complex systems often face tasks of resource distribution, including knowledge [9]. At present, the most effective means of knowledge distribution is the ontological model [11]. It should be noted that, in many of these works, the distributed ontology is not fully formalized, which does not allow us to abstract from the individual cases and solve the tasks of building datasets and knowledge in a general way. Using the distributed ontologies is advisable only when really enormous volumes of heterogeneous information exist [12]. The most characteristic feature of complex systems, including integrated ones, is decentralization and a low level of unification $[11,18,19]$. There is no technical possibility of the simultaneous processing of all system information, and it is also impossible to implement universal algorithms of knowledge processing. The information space of complex systems is not sufficiently normalized due to the qualitative and quantitative content. This space is characterized by linguistic variables that expand the space to a fuzzy one [1-3,12-14]. The ontology is a formal representation of reality; it requires the clear delivery of information, but reality cannot always be clearly expressed, so it is recommended to use fuzzy ontology [11].

The subject of ontology is the study of the categories of things that are or can exist in a particular subject area [4]. The ontology product is a catalog of types of things that are believed to exist in a certain area $D$ for a person who uses the language $L$ to describe $D$. Types of ontology are entities, meanings of words, concepts, and the relations of the language $L$ to the aspect of description $D$ [19]. Uninterpreted logics, such as the calculus of predicates [5] and conceptual graphs [6], are ontologically neutral. They do not affect the subject or how it can be interpreted. Logic itself means nothing, but the combination of logic with ontology generates a language that can express the relationship between objects in a certain subject area [7].

The existing applied solutions regarding the distributed ontologies are divided into two large groups:

1. The development of ontologies;

2. The use of ontologies.

The first group includes the development environments, including visualization environments [4], automatic integration mechanisms [13], and version control systems [14], the qualitative review of which was carried out in [5]. Using the first type involves incomplete ontology, which restricts the use of the semantic component. The second group includes information systems based on ontologies [8] and the mechanisms of logical conclusion $[15,16]$. The second group of solutions can fully use the semantic load of the ontology without restrictions.

During the development of ontologies, it is necessary to take into account the following:

- The informal ontology can be specified by a set of types, which are defined using natural language;

- The formal ontology is specified by a set of concept names and relations, which are partially ordered using the "type-subtype" relation [11].

The principal tasks that are solved during the creation of systems based on distributed ontologies are the solution of conflict in the case of a conflict and the increase in the completeness and accuracy of information searching in the ontological structure.

One of the important questions relevant to the ontological structures of complex systems is the logical conclusion based on inadequate input data due to such reasons as [15]:

- The impossibility of the constant functioning of all the subsystems which contain knowledge;

- An insufficient amount of time resources needed to obtain all the input data;

- An insufficient amount of computing resources needed to process all the input data;

- The incompleteness of the input data in the knowledge base.

\section{Materials and Methods}

The important difference between the ontological representation of knowledge and simple datasets, frame models, and semantic networks is: 
- The presence of meta-information of any order, which allows us to describe subject areas simultaneously at any level of abstraction [19];

- The minimization of data separation and their description [4];

- The simplicity of knowledge representation, with wide opportunities for automatic interpretation using unified methods [20].

Any ontology Ont is reduced to the expressiveness of descriptive logic and can be presented as a tuple:

$$
\text { Ont }=\langle T B o x, R B o x, A B o x\rangle \text {, }
$$

where TBox is the terminology hierarchy consisting of a set of concepts $c$; RBox is the relations hierarchy, consisting of a set of relations $r ; A B o x$ is the statement set $a$, based on TBox and $R B o x$; and $c$, and $r$ are ontology elements.

It is necessary to configure the process of detecting a specific change $\omega$ in ontology Ont automatically when element $E_{\text {lem }}$ changes to the variable $\xi_{i}$ :

$$
\Delta \text { Ont }=\omega \mid \forall \Delta \text { Elem }_{i} \in \text { Ont } \mid \xi_{i} \neq \varnothing .
$$

The identification of change $\omega$ significantly increases the work efficiency, reduces the cost of resources for improving the ontology development, and ensures the high reliability of managerial decisions. In order to determine the change $\omega(1)$, it is necessary to build classic models of static ontology Ont and ontology, which changes over time $t$, Ont $(t)$, and to find the regularities of these models.

The improvement of modern complex ontologies has a spontaneous and poorly formalized nature, resulting in the creation of unique structural solutions. Most often, such solutions are not optimal and require additional processing to reduce the complexity of the used algorithms [5].

Let us consider two types of ontologies development—extensive and intensive [11].

The extensive development of ontology is elementary ontology Ont $t_{e}$ for a specific ontology Ont $_{r}$ [11]. It must be taken into account that the ontologies in Ont $t_{r}$ will be denoted as Ont $t_{\text {ext }}$ and called an extended:

$$
O n t_{r}=\left\langle O n t,\left\{O n t_{e x t}\right\}\right\rangle
$$

It can be assumed that the intensive development of ontology Ont' is such an ontology Ont, which reproduces many specific statements Ont' [11].

$$
\text { Ont } \cap \text { Ont }^{\prime}=\text { Ont }_{\xi} \neq \varnothing .
$$

The proposed extensive development describes the subject area of ontology Ont $t_{r}$ fully and in detail. Intensive development shows a set of real Ont' and specifies it depending on the identified errors, taking into account the following methodological aspects:

$$
O n t_{e}=\langle K B, M I\rangle,
$$

where $K B$ is the knowledge base of ontology elements; $M I$ is the meta-information of ontology,

$$
\text { indep }(A, B)=\text { true } \mid \begin{aligned}
& A_{1}{ }^{I}=A_{2}{ }^{I}, B_{1}{ }^{I}=\Delta^{I}, B_{2}{ }^{I}=\varnothing, \\
& A_{1}, B_{1} \in \text { Ont }_{1}, \\
& A_{2}, B_{2} \in \text { Ont }_{2}, \\
& \text { Ont }_{1} / B_{1}=\text { Ont }_{2} / B_{2},
\end{aligned}
$$

where $\operatorname{indep}(A, B)$ is an independent element $A$ from element $B$ of ontology Ont; $A$ and $B$ are the ontology elements Ont; $\Delta^{I}$ is the specified universe; and $O n t_{1}$ and $O n t_{2}$ are the specific ontologies.

Let us consider an improved formal base for the extensive development of ontologies, which can be further used for constructing methods and algorithms during work with distributed ontologies. 
During this research, the noncontradictory ontologies were applied; this restriction does not reduce the effectiveness of the obtained results, but determines the decomposition level of their use. For any two ontologies, they are associated with the import relation:

$$
\operatorname{import}\left(R I^{+}, R I^{-}\right)=\left\{\begin{array}{l}
1, R I=\text { true } \\
0, R I=\text { false }
\end{array}\right.
$$

where $R I^{-}$is the basic ontology; $R I^{+}$is the ontology, which imports; $R I^{-}$and $R I^{+}$use each other as the basis to build their knowledge structure; and $R I$ is the import relation.

Creating the structure, the first ontology develops the knowledge being stored in base one Ont $t_{\text {base }}$. This is a simple development that increases knowledge (Figure 1).

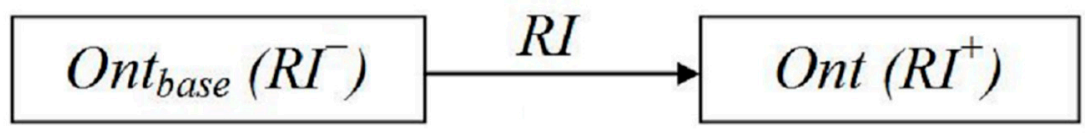

Figure 1. Simple extensive development of ontology.

Extension elements do not always depend on the base ontology Ont $t_{\text {base }}$. There are some cases when none of the extension elements depend on the base ontology.

Let us introduce the concept of pseudo-import. A pseudo-import is RI, so that:

$$
p R I=R I, \quad R I=1, \quad \text { indep }\left(R I^{-}, R I^{+}\right)=1(\text { true }),
$$

where $p R I$ is a pseudo-import.

The connection of the development of the form (2) can be removed during the stages of analysis of the general structure according to the following condition: removing some $p R I$, it is necessary to preserve the transitive integrity of the structure (Figure 2).

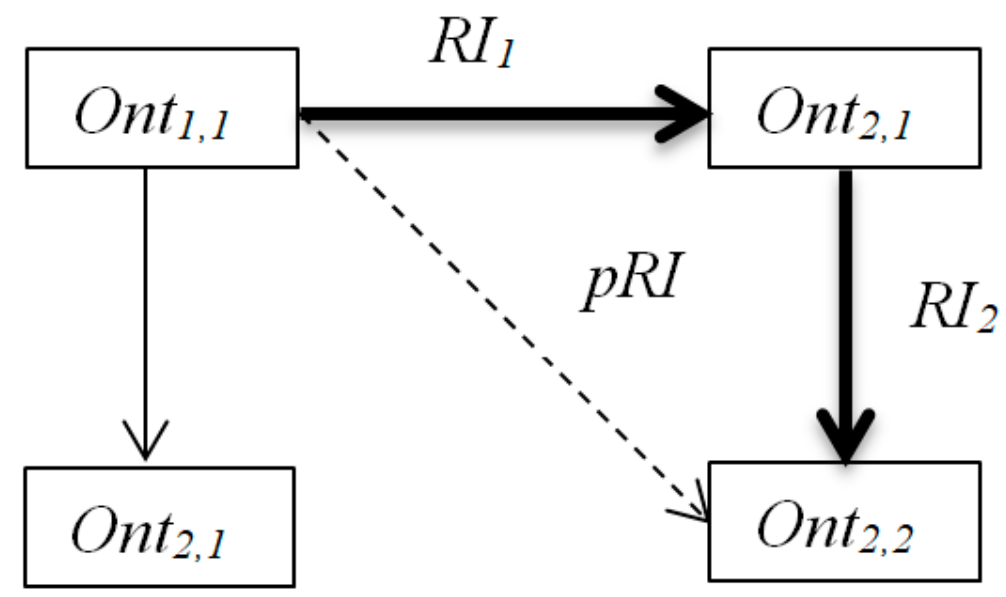

Figure 2. Fragment of the transitive integrity of the structure.

Removing the set of elements, on which ontology elements do not depend, does not influence the general semantics of the ontology. The preservation of transitive integrity during removing the relations of the form (2) is provided by redirecting the connection to all ontology extensions $R I^{-}$ ( $p R I$ is eliminated).

Let us consider the representation of the ontology development using the structure $\langle T, A\rangle$, which provides the components of ontology expansion in addition to the existing (base) ontology. 
The representation of the ontology in the form of a meta-description has a number of advantages concerning the application tasks:

- The configuration corresponds to the generalized approaches to the ontology description;

- The configuration allows us to increase the detailing of the consideration of distributed ontology, without using complex models and software tools;

- The presentation is visual;

- The presentation is used effectively in some existing application programs [12].

At a more detailed level, the simple extension of the base ontology has the following interpretation:

$$
\text { Ont }_{\text {ext }}=\left\langle T_{b}+T_{e}, A_{b}+A_{e}\right\rangle
$$

where $\left\langle T_{b}, A_{b}\right\rangle=$ Ont $_{\text {base }} ;\left\langle T_{e}, A_{e}\right\rangle$-extension elements.

In practice, the extension of the base ontology (3) is carried out through adding the axioms:

$$
\text { Ont }_{\text {ext }}=\left\langle T_{b}, A_{b}+A_{e}\right\rangle
$$

The set of all extension elements (4) can be called resource $R$. The ontology extension using resource $R$ allows us to apply the base ontology as a data schema.

The complex type of development of the ontological structure is an integration view. The possibility of integration is one of the basic requirements for ontology. It is characterized by the import of a set of base ontologies.

Formally, the integration development of ontologies has the following form (Figure 3):

$$
R I_{j}=\text { true } \mid R I_{j}^{-}=\text {Ont }, R I_{j}^{+}=\text {Ont }_{\text {base }_{j}}, \forall j=\overline{1, M},
$$

where Ont $t_{\text {base }}$ is the $j$ base ontology, Ont is the general extension for all Ont base $_{j}$, and $M$ is the number of ontologies that integrate into Ont.

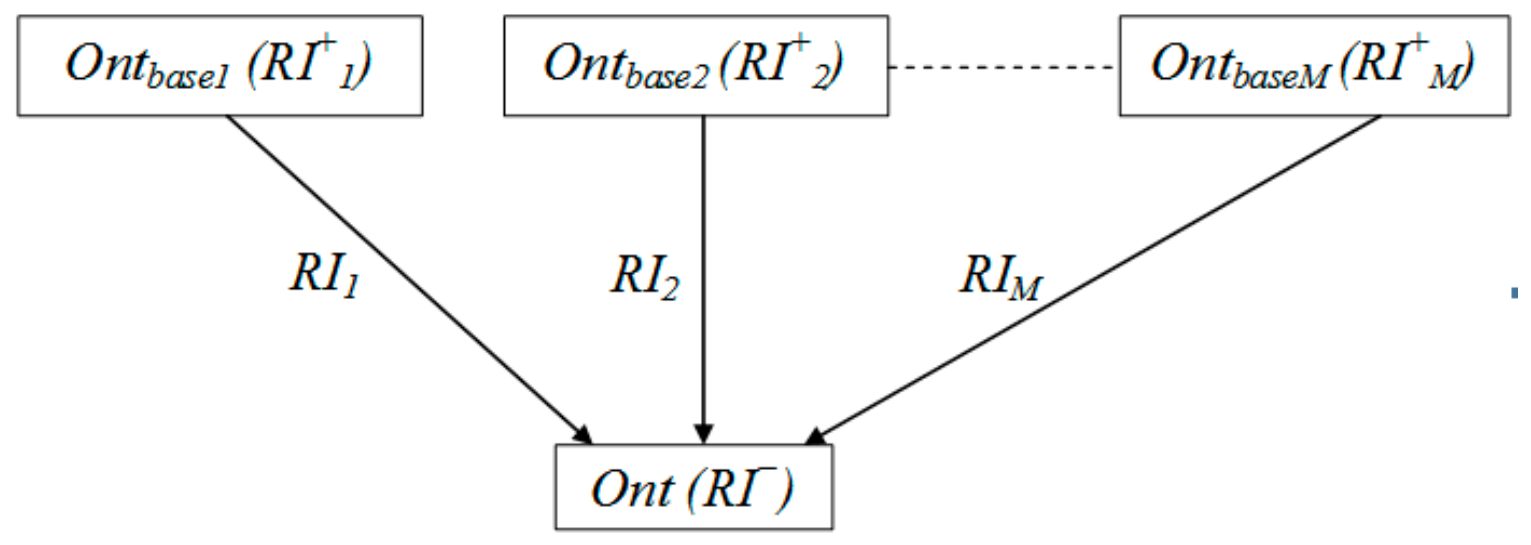

Figure 3. Integrative extensive development of ontology.

At the more detailed level, the integrating development can be represented as

$$
\text { Ont }_{e x t}=\left\langle T_{b_{1}}+\ldots+T_{b_{m}}+T_{e}, A_{b_{1}}+\cdots+A_{b_{m}}+A_{e}\right\rangle .
$$

Moreover, Ont $_{\text {base }_{j}}=\left\langle T_{b_{j}}, A_{b_{j}}\right\rangle$ is systemically consistent.

The systemically consistent ontology is the set of ontologies for which it is possible to create at least one integrating resource (5) containing at least one noncontradictory axiom. The contradictory axiom is an axiom which is equivalent to the empty set under the given conditions. 
Co-development is an important feature of ontologies (Figure 4); there is no any hierarchy, and i.e., each of the two ontologies is the base and extension for each other.

$$
\text { import }\left(\text { Ont }_{x}, \text { Ont }_{y}\right) \cap \text { import }\left(\text { Ont }_{y}, \text { Ont }_{x}\right)=1,
$$

where $O n t_{x}, \mathrm{Ont}_{y}$ are the common ontologies under development.

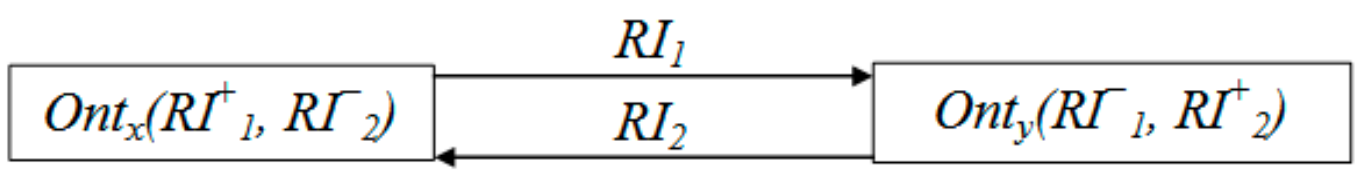

Figure 4. Common extensive development of ontology.

Spatially, there are two different ontological substructures, $O n t_{1}$ and $O n t_{2}$, but they are always semantically indivisible and are some generalized structure.

Common ontologies under development are not limited by only two substructures; they may be composed of a cycle of any length:

$$
\text { Ont }_{\text {total }}=\left\{O n t_{l}\right\}, \forall l=\overline{1, L}
$$

where Ont total is the general ontological structure; $O n t_{l}$ is an element belonging to the closed import path; $L$ is the overall length of the cycle; and:

$$
\begin{aligned}
& \text { import }\left(\text { Ont }_{i}, \text { Ont }_{i+1}\right)=1, \quad \forall i=\overline{1, L-1}, \\
& \text { import }\left(\text { Ont }_{L}, \text { Ont }_{1}\right)=1 .
\end{aligned}
$$

A certain extension Ont of the ontological structure is included in some common development Ont total (6), when import (Ont, Ont) $=1$-i.e., Ont is the base for itself.

The improvement of the hybrid development of ontologies contains all these features simultaneously.

Today, in the aspect of the consideration of distributed ontologies, there is a task associated with working with fuzzy imports.

The fuzzy import $\widetilde{R} I m p o r t$ is the import RImport for which the membership function is defined [3,11], which is interpreted as a coefficient of confidence in the existence of the precise RImport:

$$
\widetilde{R} I m p o r t=\left\langle\text { RImport }, \mu_{\text {RImport }}\right\rangle .
$$

The direct introduction of connections of the form (7) is not typical during the creation of distributed ontologies. The fuzzy import can be detected during the analysis of the structure to determine the presence of pseudo-imports.

The membership function $\mu_{\text {RImport }}$ of (7) is the maximum value of membership function $\mu(X, Y)$ :

$$
\mu_{\text {RImport }}=\mu_{\text {RImport }}\left(R I^{-}, R I^{+}\right)=\max _{i}\left(\max _{j}\left(\mu\left(X_{i}, Y_{j}\right)\right)\right)
$$

where $R I^{-}=\left\{X_{i}\right\}, R I^{+}=\left\{X_{j}\right\} ; \mu(X, Y)$ is the membership function of some relation between $X, Y$.

If at least one of the elements $X$ has the explicit reference to Ont $t_{\text {base, }}$, then this is the precise import. If all the links are fuzzy, then the confidence in this import corresponds to the maximum confidence in the existence of one of these links. 


\section{Results}

The membership functions of transitive relations can be calculated in the following way [15]:

$$
\mu_{R}(x, z)=\underset{y}{\operatorname{MAX}}\left(\operatorname{MIN}\left(\mu_{R}(x, y), \mu_{R}(y, z)\right)\right),
$$

or

$$
\mu_{R I}\left(\text { Ont }_{1}, \text { Ont }_{3}\right) \underset{\text { Ont }_{2}}{\operatorname{MAX}}\left(\operatorname{MIN}\left(\mu_{R I}\left(\mathrm{Ont}_{1}, \mathrm{Ont}_{2}\right), \mu_{R I}\left(\mathrm{Ont}_{2}, \mathrm{Ont}_{3}\right)\right)\right) .
$$

To solve the task in general,

$$
\mu_{R I}(\text { Ont }, \text { Ont })=1 \text {. }
$$

The import in fuzzy ontological structures has some features which take into account (6).

The incoherence of fuzzy sets is expressed in the fuzzy ontology through the function incoher $(U, V) \in[0,1]$, then:

$$
\begin{gathered}
\text { Ont }=\bigcup_{i} \text { Ont }_{i} \bigcap_{i} \bigcap_{j} \text { incoher }\left(\text { Ont }_{i}, \text { Ont }_{j}\right)=\alpha, \\
\forall i, j=\overline{1, N}, i \neq j,
\end{gathered}
$$

where $\alpha \in[0,1]$ is the threshold level of insecurity that is specified by the subject domain expert.

We can obtain the improved model for the extensive development of fuzzy ontologies and some basic relations. This model is based on the relations of ontology import and on the property of the independence of ontology elements. This model allows us to describe the static distributed ontologies in general terms. Its use allows us to analyze and modify existing structures algorithmically.

It is necessary to improve the model of the presentation of intensive ontological development, which gives us the opportunity to create effective methods for intelligent systems of decision support during solving ontological engineering tasks.

Ontology Ont is represented by a set:

$$
\text { Ont }=\langle M O, M R I\rangle
$$

where $M O=\left\{O n t_{i}\right\}$ is the set of elementary ontologies with a tendency to discrete changes (these ontology changes are in intensive development); $M R I=\left\{R I_{j}\right\}$ is the set of all import relations $R I$ specified on the base of ontology.

The section "Results" is based on some results obtained in [11], so the intermediate results are not presented in this article.

It can be assumed that the ontology elements Ont may tend to change over time. The changes are discrete and are made in accordance with the syntax of the chosen language for representing ontologies. Such ontology changes can be called an intensive development.

Any ontology Ont can be changed over time only in a discrete way. The intensive development is a tuple $D$ which describes the evolution Ont: a specific ontology and the moment of time of its obtaining.

$$
D=\left\{\left\langle O n t_{i}, t_{i}\right\rangle_{i}\right\} \mid t_{i}<t_{i+1}, \quad i=\overline{0, K},
$$

where $K$ is the final nonnegative version number Ont.

The improved development of ontology has the following form:

$$
R D \Rightarrow\left\langle i n c \_N, d e c \_N, i n c \_E, d e c \_E, c h \_E\right\rangle,
$$

where $i n c_{-} N$, dec_N is the set of dependent connections, $i n c_{-} E$, dec_E is the set of independent connections, and $c h \_E$ is the set of changed connections ( $c h \_E=\varnothing$ for the precise ontology). 
The improved model of the intensive development of ontology was obtained. This model takes into account the discreteness of ontological knowledge and allows us to represent the ontology evolution process over time.

Let us consider the distributed ontology (8).

It can be assumed that any $O n t_{i} \in M O$ corresponds to some development $D_{i}(9)$. For any time value $t$, we can build the corresponding import structure $M R I(t)\left(M R I=\left\{R I_{i}\right\}, i=\overline{1, N}\right)$, presented in Figure 5 , where the straight line represents $R I$ (import relation), the dashed line represents $n R I$ (constructed relation), and the smaller dashed line (shown by $D_{1}$ and $D_{2}$ ) represents $R D$ (version relation).

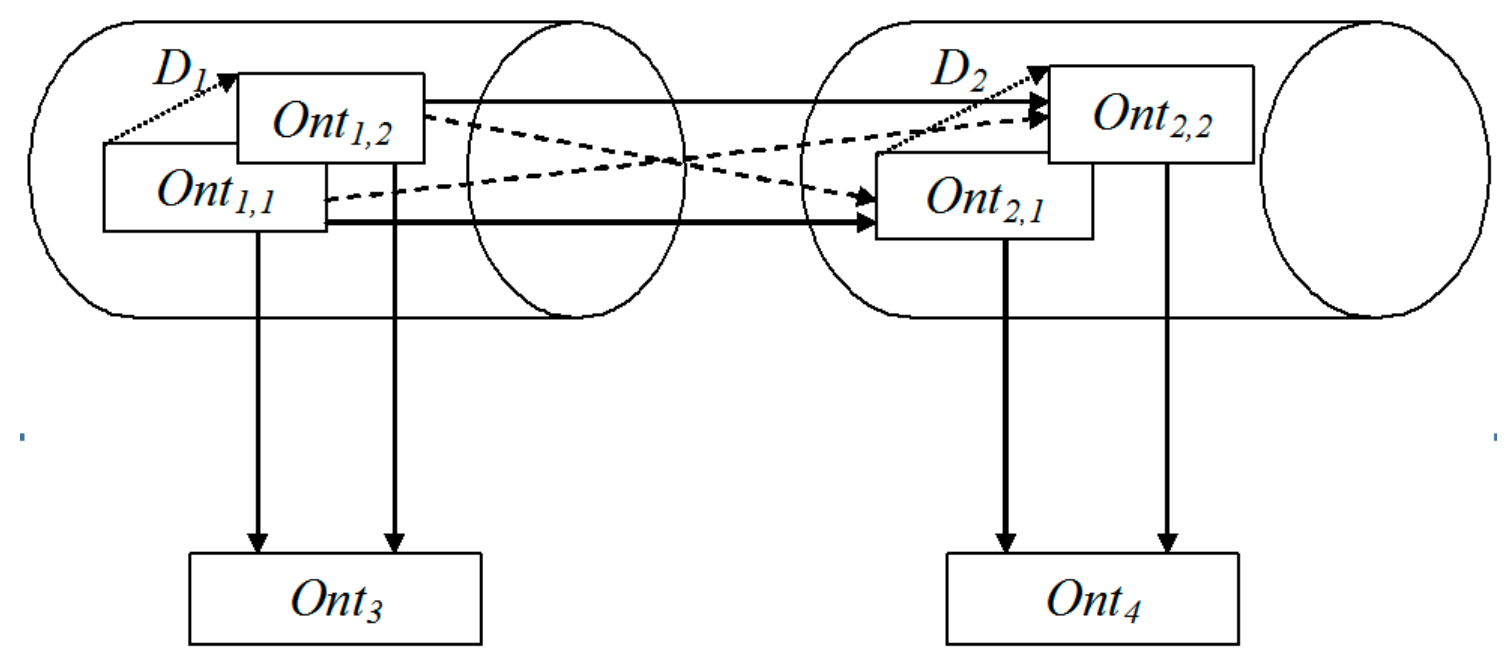

Figure 5. Scheme of the advanced hybrid development of ontology.

Under the condition $M R I(t)=M R I$, where $M R I$ is a constant, we observe the process of intensive change under the static state of the extensive structure. When there is not any change in the structure of import during development, we can consider the change in the entire ontology as a set of intensive parallel processes providing the individual ontologies.

The improved hybrid model of ontology development may contain gaps in the functions of the ontology values. It can be assumed that some ontology Ont is replaced by two (or more) ontologies $O n t_{1}$ and $O n t_{2}$ during evolution, and $O n t=O n t_{1} \cup O n t_{2}$.

Taking into account the continuous value of proximity, we obtain the following: aboutness $($ Ont, Ont 1$) \cap$ aboutness $\left(\right.$ Ont $_{1}$ Ont $\left.t_{2}\right)=$ false, as well as aboutness $\left(\right.$ Ont, Ont $_{1} \cup$ Ont $\left._{2}\right)=$ true. $_{\text {. }}$ There is a gap in the $D$ value and the occurrence of developments $D_{1}$ and $D_{2}$.

$$
D \rightarrow D_{1}, D_{2} \text { or } D_{1}, D_{2} \rightarrow D
$$

An improved ontology development model is:

$$
\operatorname{Ont}(t)=\left\langle\left\{f_{D_{i}}(t)\right\}, M R I(t)\right\rangle .
$$

Generalized intensive development:

$$
D=\left\{\left\langle\left\{\text { Ont }_{j}\right\}_{i}, t_{i}\right\rangle_{i}\right\} \mid t_{i}<t_{i+1}, \quad i=\overline{0, K}, \quad j=\overline{1, J(t)},
$$

where $J(t)$ is number of ontologies at time $t$ related to development $D$.

The generalization obtained:

$$
\left\{O n t_{j}\right\}_{i}=f_{D}(t) \mid \forall t, t \in\left[t_{i}, t_{i+1}\right),
$$


where $f_{D}(t)$ is the function of the ontology value at time $t$ for development $D$.

As a result, we obtain the improved hybrid model of (extensively intensive) ontology development, which describes adequately the evolution of any level of complexity in space and in time.

Thus, it obtains the hybrid mathematical model of ontology development, which integrates extensive and intensive models. The improved model allows us to solve different tasks effectively, to analyze and evaluate the space of states of the discussed ontologies, and to identify and eliminate the properties of inadequacy (incompleteness, redundancy, and inconsistency [22]). This model is based on the discreteness of ontology changes over time, as well as on the concepts of independence of elements and ontology imports. The presented model adequately describes the development of precise and fuzzy ontologies.

\section{Implementation of the Method of Eliminating the Inadequacy Properties}

Ontologies are complex objects; their life cycle includes many stages of improvement. Monitoring and evaluating the changes that occur during their evolution is a complex task.

The method for determining and eliminating the properties of inadequacy of ontology development is implemented as follows:

- Choose the elementary ontology Ont and create the ontology Ont by means of meta-information using the import relation import $\left(R I^{+}, R I^{-}\right)=\left\{\begin{array}{l}1, R I=\text { true, } \\ 0, R I=\text { false. }\end{array}\right.$

- Define the stages of development Ont within which the analysis of adequacy is carried out. The structure Ont can be supplemented with the ontologies which stopped importing over time.

- On the basis of the available data, build the most complete model of hybrid extensively intensive development $\operatorname{Ont}(t)$. In some cases, the constructed model $\operatorname{Ont}(t)$ is the simple model of extensive or intensive development.

- After obtaining the formal development model Ont $(t)$, determine the values of the inadequacy criteria-namely, incompleteness ( $\mathrm{Npl}$ ) and redundancy (Iz). The result of determining these criteria can be effectively interpreted into a binary response.

- Find and eliminate the inadequate properties according to the method of determining and eliminating the inadequate properties during the ontology evolution. Within the framework of development $\operatorname{On} t(t)$, it is necessary to find the adequate subsets of changes or to affect the model in order to maximize the overall level of adequacy;

- The method of determining and eliminating the inadequacy in ontology development is implemented through an algorithm that provides the search of the upper limit of an adequate state.

Fixing some time of ontology development $t$ Ont, the inadequacy of the structure was detected. The use of previous versions of ontologies associated with Ont may result in the adequacy of the entire structure.

Let us consider the algorithm for finding the upper limit of the adequacy of ontology development (Figure 6).

It can be assumed that there is development $\operatorname{Ont}(t)=\left\langle\left\{f_{D_{i}}(t)\right\}, M R I(t)\right\rangle$, for which it is necessary to determine all the upper limits of adequacy for the ontology Ont at a given moment $t_{k}$.

Step 1. Set the input values input ontology Ont and elementary ontology Ont $t_{e}$ with Ont, for which the search $\left(D_{\text {ont }}=\langle O n t, t\rangle\right)$ is carried out. Add Ont to the structure queue Queue.

Step 2. The current Ont is equal to the first value in the queue Queue. Remove the first value from the Queue.

Step 3. Check the inadequacy $N p l\left(\mathrm{Ont}_{i}, \mathrm{Ont}_{i-1}\right)=\alpha^{N p l}{ }_{i} \mid \forall \mathrm{Ont}_{i} \in \mathrm{Ont}(t), \alpha^{N p l_{i}} \in[0,1]$ (criteria for detecting incompleteness $\mathrm{Npl}$ ) of the current state Ont: $n$ Adequacy $(\operatorname{Ont}(t))$; if $n$ Adequacy $(\operatorname{Ont}(t))=$ true, then go to Step 4, otherwise go to Step 7. 
Step 4. Perform the rollback operation (the operation of obtaining the previous versions of the ontology):

$$
\operatorname{Rollback}\left(f_{D}\left(t_{1}\right)\right)=f_{D}\left(t_{2}\right),
$$

where $\left.t_{2}<t_{1}, t_{2}<t_{1}, \forall t, t<t_{1}, f_{D}\left(t_{1}\right)\right) \neq f_{D}(t)$ for a set of elementary ontologies of the current structure Rollback $($ Ont $)=\left\{\right.$ Ont $\left._{i}\right\}$.

Step 5. Check whether any values Ont were not in the queue before. Add every $\left\{\mathrm{Ont}_{i}\right\}$ to the queue Queue.

Step 6. Go to Step 2.

Step 7. Add the current Ont to the resulting list "Resulting list".

Step 8. If the Queue is not empty, then go to Step 2, otherwise Exit.

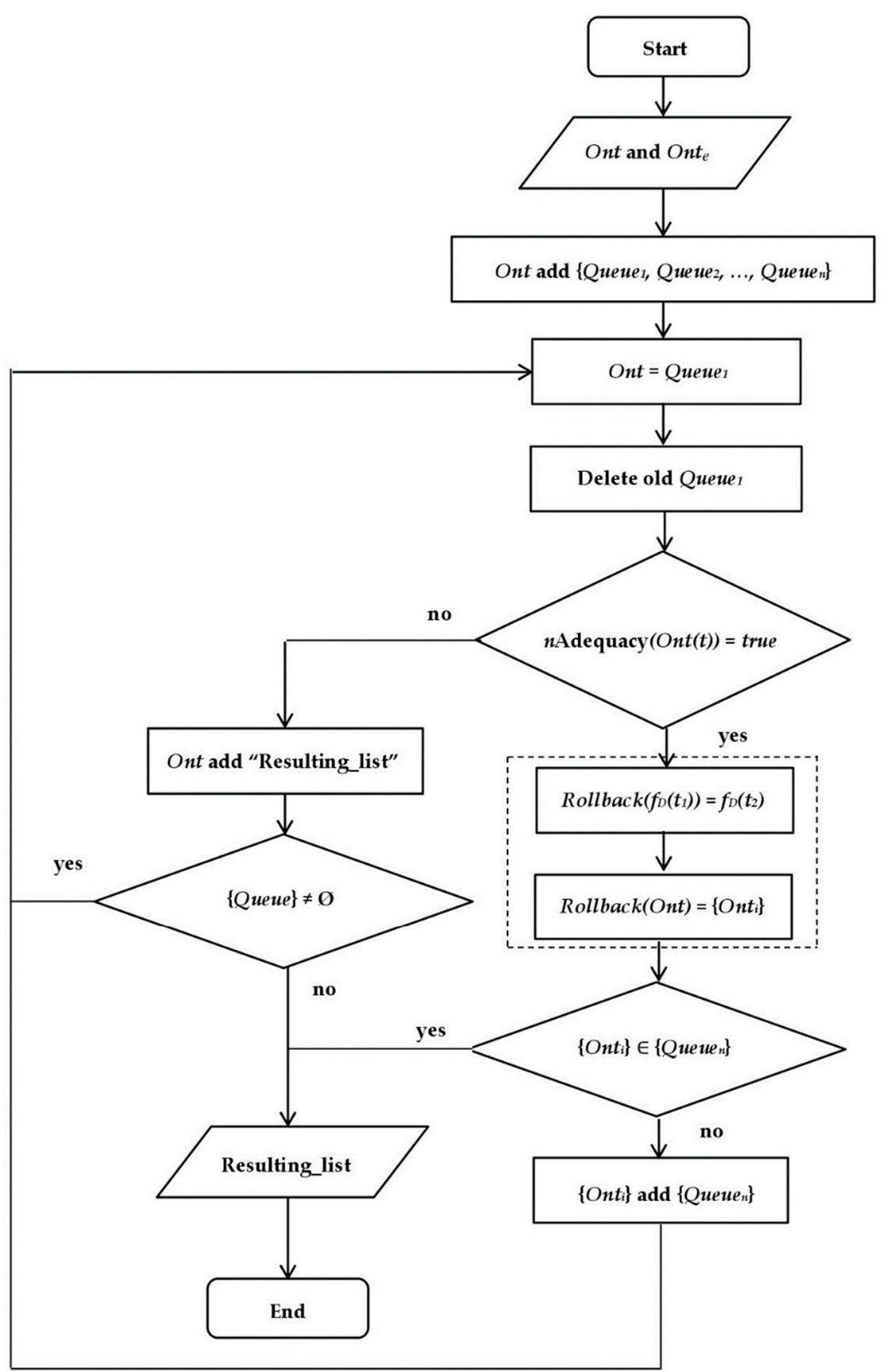

Figure 6. Flow chart of searching the upper limit of adequacy of ontology development. 
During the software implementation of this method, it is necessary to fix and take into account the value of the membership function of ontologies $k$ to the class of consistent ones:

$$
k=\max \left[\max [\operatorname{avg}(N p l), \operatorname{avg}(I z)]-d e f_{c h}, 0\right],
$$

where $\operatorname{avg}(\mathrm{Npl}), \operatorname{avg}(\mathrm{Iz})$ are the average values of adequate criteria (redundancy and incompleteness, respectively); $d e f_{c h}$ is the configurable generalized threshold of acceptable changes.

The development of fuzzy ontology is adequate if the value is $A \geq 0.5$.

The ontological representation of knowledge is associated with significant requirements for computational resources-namely, memory $v$ and computation time $t$. In this regard, any algorithmic solutions in this area require testing for computational complexity.

For the constructed models of ontology development, there are some proposed recommendations for application which allow us to implement software solutions for the simulation and analysis of the ontology evolution processes. To solve the practical tasks, there are some represented and well-grounded algorithmic tools for analyzing the researched ontologies using generalized criteria of inadequacy within the developed hybrid model of the development of fuzzy ontologies.

\section{Experiments and Discussion}

Let us consider the application of the structural-temporal approach to the Semantic Web for Earth and Environmental Terminology (SWEET) ontologies [23].

The SWEET NASA ontology is the generalized ontology that describes the basic mathematical, physical, chemical, biological, astronomical, and geographical phenomena; human activities; and some specific knowledge. Its dimension is about 7200 elements (4500 classes, 200 relations, and 2500 objects).

SWEET ontologies are hybrid extensively intensive development (the structure of imports is preserved over a certain period of time).

The process of the simplification of the extensively intensive development is shown on the ontology with 180 classes, 270 relations, and 3950 objects.

It can be assumed that a certain set of ontologies is imported into Ont:

units, foaf, $r d f$, xsd, $r d f s$, owl, wot, dc, dcterms, dctype, bibtex.

According to import $\left(R I^{+}, R I^{-}\right)=\left\{\begin{array}{l}1, R I=\text { true, } \\ 0, R I=\text { false, }\end{array}\right.$ we can write: import (Ont, $\{$ units, foaf $, r d f, x s d, r d f s$, owl, wot, dc, dcterms, dctype, bibtex $\})=1$.

Analyzing the connections of each of the imported ontologies, we may construct the ontological structure in accordance with (8) in the form of a graph of connections of each imported ontology (Figure 7). The resulting graph requires further simplification.

Let us find the value $p R I$ for Ont in accordance with (2):

$$
\begin{aligned}
& p R I_{1}=\text { import }(\text { Ont }, \text { bibtex }) \\
& p R I_{2}=\text { import }(\text { Ont }, \text { units }) \\
& p R I_{3}=\text { import }(\text { Ont }, \text { dctype })
\end{aligned}
$$

The vertices of the graph bibtex, units, dctype have only input arcs, but the main vertex is Ont. After the operation of folding the cycles, the new vertices are formed on the graph, and then we repeat the operation until it makes changes to the graph. Having performed the operation of the definition of a set of imports without duplicate arcs, and having applied the improved hybrid model of ontology development, we obtain the partial simplification of the structure (Figure 8a). The complete simplification of the given structure is shown in Figure 8b. 
The resulting structure contains an exclusively additive type of development which is the simplest. Obviously, it is much simpler than the original structure.

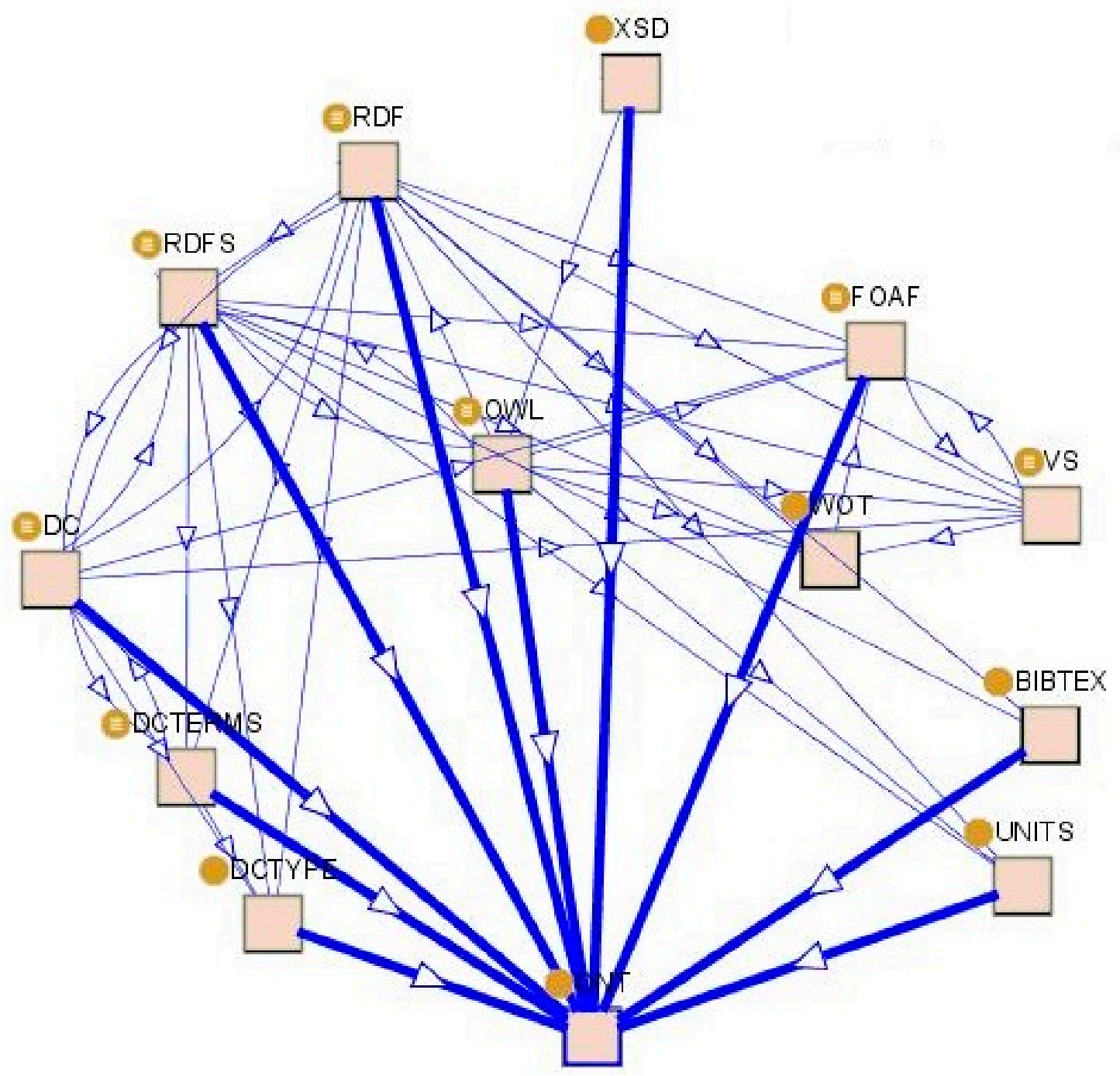

Figure 7. Structure of the nodes and import of ontologies.

Let us demonstrate the process of the intensive development of the basic elementary ontology "units" with SWEET. This ontology contains information about physical units of measurement. The study was conducted in three versions:

$$
D_{\text {units }}=\left\langle\left\{\text { units }_{1}, t_{1}\right\},\left\{\text { units }_{2}, t_{2}\right\},\left\{\text { units }_{3}, t_{3}\right\}\right\rangle .
$$

The transition from version 1 to version 2 was caused by the requirement to add basic proportions-for example, "percentage" and "ppm". The change in version included the change in the representation of the "volt" concept. The set of added objects is reflected by the quantitative characteristics of the VERSION 12 line (Table 1).

The transition from version 2 to version 3 was accompanied by fewer changes, but they were more complex. There are a number of new relations between existing objects. Some string values have been corrected. The complex process of change is represented in column A and in row VERSION 23 (Table 1).

Obviously, VERSION $_{13}$ line is equivalent to the elemental addition of lines VERSION 12 and VERSION $_{23}$. This confirms the linearity of development $D_{\text {units }}$ as well as the independence of the changes. 
The software implementation of the program for the integration and presentation of heterogeneous data of information systems is carried out using the method of determining and eliminating the properties of the inadequacy of ontology development.

For development (10), Table 2 presents the detailed results.

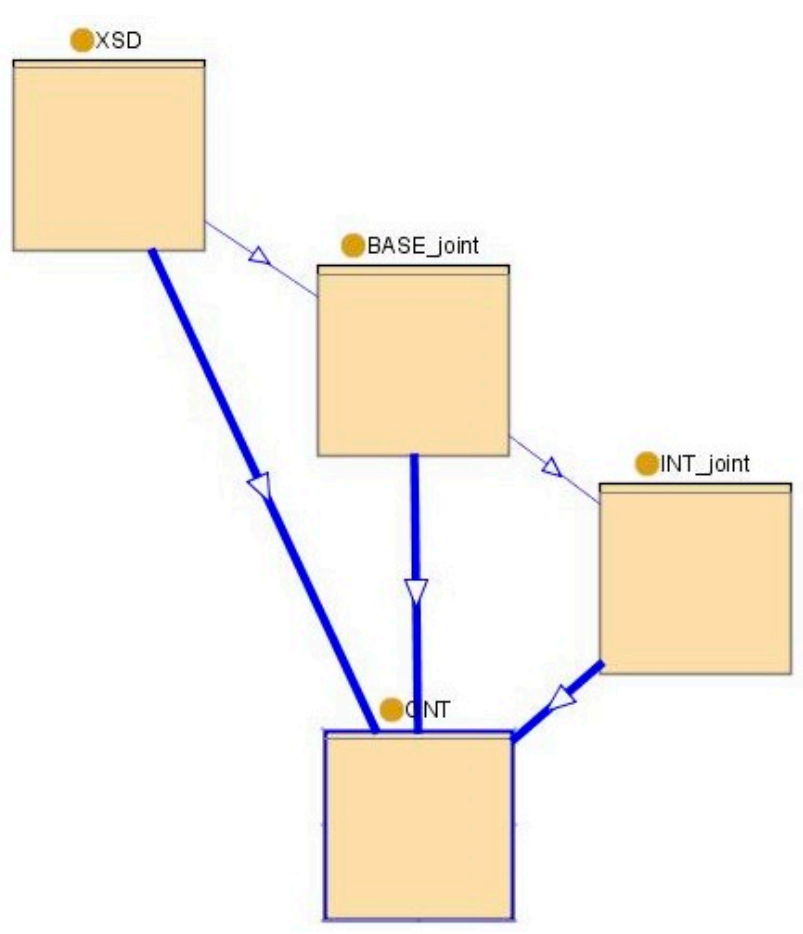

(a)

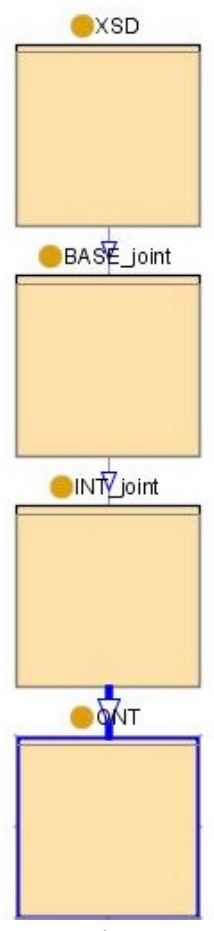

(b)

Figure 8. Simplified node structure and ontology import: (a) partial simplification, (b) full simplification.

Table 1. Development tuples of subgraphs of the "units" ontology.

\begin{tabular}{cccc}
\hline Version & T & TA & A \\
\hline VERSION $_{12}$ & $<0,0,0,0,0>$ & $<13 \mathrm{~N}+91 \mathrm{E}, 0,0,0,0>$ & $<13 \mathrm{~N}+14 \mathrm{E}, 1 \mathrm{~N}+1 \mathrm{E}, 0,0,0>$ \\
VERSION $_{23}$ & $<0,0,0,0,0>$ & $<1 \mathrm{~N}+10 \mathrm{E}, 0,0,0,0>$ & $<3 \mathrm{~N}+4 \mathrm{E}, 1 \mathrm{~N}+1 \mathrm{E}, 2 \mathrm{E}, 1 \mathrm{E}, 0>$ \\
VERSION $_{13}$ & $<0,0,0,0,0>$ & $<14 \mathrm{~N}+101 \mathrm{E}, 0,0,0,0>$ & $<16 \mathrm{~N}+18 \mathrm{E}, 2 \mathrm{~N}+2 \mathrm{E}, 2 \mathrm{E}, 1 \mathrm{E}, 0>$ \\
\hline
\end{tabular}

Table 2. Values of criteria $\mathrm{Npl}$ and $\mathrm{Iz}$ for development (10).

\begin{tabular}{cccc}
\hline Ontology & Criterion & Max & Avg \\
\hline \multirow{2}{*}{ units } & $N p l$ & 1 & 0.03 \\
& $I z$ & 1 & 0.05 \\
\hline
\end{tabular}

The value of the function of the membership of the ontology development relates to the class of consistent $k=0.05$.

The ontology development is adequate; the value $A=0.66$ meets the accepted condition $A \geq 0.5$.

The user interface for monitoring the adequacy of ontology development with preliminary automatic preparation and analysis is shown in Figure 9. 


\section{CONTROL ADEQUACY OF ONTOLOGY DEVELOPMENT}

Development is adequate

- $k=0.05$

- $\mathrm{Npl}=0.03$

- $\mathrm{Iz}=0.05$

In detail $\gg$

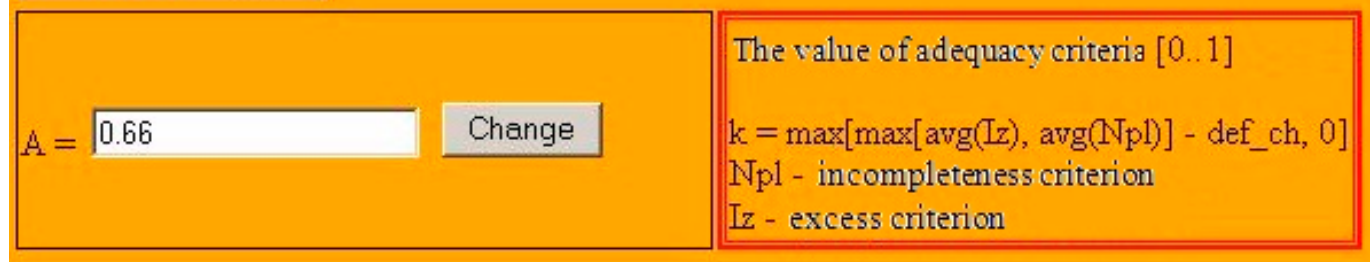

Figure 9. Interface for monitoring the adequacy of ontology development.

This development is hybrid intensively extensive, with a constant structure of imports, and provides a decrease in the number of objects, which are duplicated, and an increase in the semantic connections between research objects.

The models of the extensive and intensive development of ontologies allow us to divide the analyzed object into elementary modules, which gives us the opportunity to operate with a limited amount of data per unit of time.

The analysis of the RAM consumption for the proposed models (Table 3) showed that the consumption linearly depends on the number of elements that make up the structure module being considered at the moment.

Table 3. Consumption of RAM depending on the dimension of the ontology.

\begin{tabular}{ccc}
\hline |Ont $\mid$ & Min, $\mathbf{M b}$ & Max, $\mathbf{M b}$ \\
\hline 10 & 0.1 & 0.2 \\
50 & 0.6 & 1.2 \\
100 & 1.3 & 2.5 \\
200 & 2.65 & 5.1 \\
1000 & 15.2 & 23.8 \\
10,000 & 161 & 230 \\
\hline
\end{tabular}

For the number of elements in the ontology-10,000, which is a large segment of development-you need a maximum of $230 \mathrm{Mb}$ of RAM; this allows us to build models on the most modern personal computers. There is no need to impose the additional requirements for software implementations and hardware.

Thus, the method of the effective use of the fuzzy ontology development model in intelligent decision-making systems was implemented using the Borland Delphi software. The task of eliminating inadequacy (incompleteness, redundancy, inconsistency), which occurs in the course of ontology development, has been solved. The algorithmic and decision-making tools have been introduced during the management of integration processes and the support of complex system ontologies.

\section{Conclusions}

Models of the extensive and intensive development of ontologies were proposed; these models take into account the description of ontologies in space and time. This interpretation allows us to 
solve problems regarding the space of states of ontologies under development to find and remove the properties of inadequacy using the mechanisms of computational intelligence.

The formal model of hybrid (extensively intensive) development has been improved, which includes the resulting models of the extensive and intensive development of ontologies. The model allows us to consider any ontological evolution in a general way and to cover fully the life cycle of ontology, which is necessary during the design and construction of the management system of the distributed ontology. The work reveals the features of the application of fuzzy ontologies in terms of the relations of fuzzy ontology import that are sometimes important for solving complex structured problems.

The presented models provide the integration of a set of ontologies using the relations of import, which significantly reduces the time resources for generalizing the conclusions of the individual ontologies under development by means of computational intelligence.

The experimental operation of the proposed software application based on the model of the hybrid extensively intensive development of ontologies has confirmed the effectiveness of the integration and presentation of the heterogeneous data of information systems during decision-making regarding the objects of the subject area.

The requirements for the amount of memory for the implementation of the proposed models are commensurate with the size of the ontology description, and the analysis time is much less than the process of direct ontology loading.

The further stages of research in this direction may be the classification of cases of the internal inconsistency of individual elements of the ontology, the improvement of functional algorithms for simplifying ontologies, the determination of evaluation criteria for ontology connectivity, and the calculation of thresholds for the fuzzy functions. It is possible to develop methods for presenting fuzzy information in ontologies, which allow us to work with fuzzy ontologies without defined specifications, significantly reducing the time resources needed for generalizing conclusions and reducing the requirements for input information.

Author Contributions: I.T. is the leader of this research. She proposed the basic idea and participated in the discussion. Y.I.D. participated in the discussion and development associated with this research. I.T. developed the implemented software for the application for the integration and presentation of heterogeneous data. I.T. and Y.I.D. were developed the algorithms and conducted the experiments. Y.I.D. participated in the research discussion and experimental design. I.T. wrote and revised the manuscript. I.T. and Y.I.D. conducted the experiments of the consumption of RAM for the proposed models analyzed. All the authors have read and agreed to the published version of the manuscript.

Funding: The research was funded at its own expense.

Conflicts of Interest: The authors declare no conflict of interest.

\section{References}

1. Saleena, B.; Srivatsa, S.K. Using concept similarity in cross ontology for adaptive e-Learning systems. J. King Saud Univ. Comput. Inf. Sci. 2015, 27, 1-12. [CrossRef]

2. Sarwar, S.; García-Castro, R.; Qayyum, Z.; Safyan, M.; Munir, F.; Iqbal, M. Ontology Based e-Learning Systems: A Step towards Adaptive Content Recommendation. Int. J. Inf. Educ. Technol. 2018, 8, 700-705. [CrossRef]

3. Mezei, J.; Wikström, R.; Carlsson, C. Aggregating linguistic expert knowledge in type-2 fuzzy ontologies. Appl. Soft Comput. 2015, 35, 911-920. [CrossRef]

4. Ruy, F.B.; Guizzardi, G.; Falbo, R.A.; Reginato, C.C.; Santos, V.A. From reference ontologies to ontology patterns and back. Data Knowl. Eng. 2017, 109, 41-69. [CrossRef]

5. Riali, I.; Fareh, M.; Bouarfa, H. Fuzzy Probabilistic Ontology Approach: A Hybrid Model for Handling Uncertain Knowledge in Ontologies. Int. J. Semant. Web Inf. Syst. 2019, 15, 1-20. [CrossRef]

6. Donalds, C.; Osei-Bryson, K.M. Toward a cybercrime classification ontology: A knowledge-based approach. Comput. Hum. Behav. 2019, 92, 403-418. [CrossRef] 
7. Rani, M.; Srivastava, K.V.; Vyas, O.P. An ontological learning management system. Comput. Appl. Eng. Educ. 2016, 24, 706-722. [CrossRef]

8. Tambe, S.S.; Kadam, G.V. An Efficient framework for E-Learning Recommendation system using fuzzy Logic and Ontology. Int. Res. J. Eng. Technol. 2016, 3, 2062-2067.

9. Guizzardi, G.; Wagner, G.; Almeida, J.P.A.; Guizzardi, R.S.S. Towards ontological foundations for conceptual modeling: The Unified Foundational Ontology (UFO) story. Appl. Ontol. 2015, 10, 259-271. [CrossRef]

10. Daradkeh, Y.I.; Tvoroshenko, I. Technologies for Making Reliable Decisions on a Variety of Effective Factors using Fuzzy Logic. Int. J. Adv. Comput. Sci. Appl. 2020, 11, 43-50. [CrossRef]

11. Tvoroshenko, I.; Ahmad, M.; Ayaz, M.; Syed, K.; Lyashenko, V.; Alharbi Adel, R. Modification of Models Intensive Development Ontologies by Fuzzy Logic. Int. J. Emerg. Trends Eng. Res. 2020, 8, 939-944. [CrossRef]

12. Tvoroshenko, I.S.; Gorokhovatsky, V.O. Effective tuning of membership function parameters in fuzzy systems based on multi-valued interval logic. Telecommun. Radio Eng. 2020, 79, 149-163. Available online: http://www. dl.begellhouse.com/ru/journals/0632a9d54950b268,1936136c5c0473c5,69ecb12d3bc3f485.html (accessed on 20 August 2020). [CrossRef]

13. Tvoroshenko, I.S.; Gorokhovatsky, V.O. Modification of the branch and bound method to determine the extremes of membership functions in fuzzy intelligent systems. Telecommun. Radio Eng. 2019, 78, 1857-1868. Available online: http://www.dl.begellhouse.com/ru/journals/0632a9d54950b268,55c1ca0a73cc00be,339e67477ec4f851.html (accessed on 24 August 2020). [CrossRef]

14. Lyashenko, V.; Mustafa, S.K.; Tvoroshenko, I.; Ahmad, M.A. Methods of Using Fuzzy Interval Logic During Processing of Space States of Complex Biophysical Objects. Int. J. Emerg. Trends Eng. Res. 2020, 8, 372-377. [CrossRef]

15. Tvoroshenko, I.S.; Gorokhovatsky, V.O. Intelligent classification of biophysical system states using fuzzy interval logic. Telecommun. Radio Eng. 2019, 78, 1303-1315. [CrossRef]

16. Ahmad, M.A.; Tvoroshenko, I.; Baker, J.H.; Lyashenko, V. Modeling the Structure of Intellectual Means of Decision-Making Using a System-Oriented NFO Approach. Int. J. Emerg. Trends Eng. Res. 2019, 7, 460-465. [CrossRef]

17. Khader, M.H.; Mohammed, A.; Mamoun, A. Structural damage assessment criteria for reinforced concrete buildings by using a Fuzzy Analytic Hierarchy process. Undergr. Space 2018, 3, 243-249. [CrossRef]

18. Nardi, J.C.; de Almeida Falbo, R.; Almeida, J.P.A.; Guizzardi, G.; Pires, L.F.; van Sinderen, M.J.; Guarino, N.; Fonseca, C.M. A commitment-based reference ontology for services. Inf. Syst. 2015, 54, 263-288. [CrossRef]

19. Verdonck, M.; Gailly, F.; Pergl, R.; Guizzardi, G.; Martins, B.; Pastor, O. Comparing traditional conceptual modeling with ontology-driven conceptual modeling: An empirical study. Inf. Syst. 2019, 81, 92-103. [CrossRef]

20. Sales, T.P.; Guizzardi, G. Ontological anti-patterns: Empirically uncovered error-prone structures in ontology-driven conceptual models. Data Knowl. Eng. 2015, 99, 72-104. [CrossRef]

21. Troullinou, G.; Kondylakis, H.; Daskalaki, E.; Plexousakis, D. Ontology understanding without tears: The summarization approach. Semant. Web 2017, 8, 797-815. [CrossRef]

22. Färber, M.; Bartscherer, F.; Menne, C.; Rettinger, A. Linked data quality of DBpedia, Freebase, OpenCyc, Wikidata, and YAGO. Semant. Web 2018, 9, 77-129. [CrossRef]

23. Beniaminov, E.M. Ontology Libraries on the Web: Status and Prospects. Autom. Doc. Math. Linguist. 2018, 52, 117-120. [CrossRef]

(C) 2020 by the authors. Licensee MDPI, Basel, Switzerland. This article is an open access article distributed under the terms and conditions of the Creative Commons Attribution (CC BY) license (http://creativecommons.org/licenses/by/4.0/). 